Polymer Journal, Vol. 6, No. 1, pp 61-71 (1974)

\title{
Network Theory for Nonlinear Viscoelasticity
}

\author{
Yo TAKano \\ Department of Physics, Faculty of General Study, Gunma \\ University, Maebashi, Japan.
}

(Received August 13, 1973)

\begin{abstract}
The network theory developed by Yamamoto (J. Phys. Soc. Japan, 11, 413 (1956)) is applied to explain at least qualitatively some of the nonlinear viscoelastic behavior of concentrated polymer systems in shearing flow, with the assumption that the probability of chain-breakage is proportional to the square of the end-to-end distance in the chain. It is shown that the shear-rate dependence of the steady viscosity is similar to the frequency dependence of the absolute value of the complex viscosity. The so-called stress overshoot at the beginning of shearing flow, the stress relaxation after the stoppage of flow, the ordinary stress relaxation under large deformation, and the superposition of a small oscillation upon steady shearing flow are treated; the results are in good qualitative agreement with the experiment. The rate-dependent and the deformation-dependent relaxation spectra are derived from the time dependence of the stresses in the two kinds of stress relaxation.
\end{abstract}

KEY WORDS Network Theory / Nonlinear / Viscoelasticity / Shear

Flow / Concentrated Polymer System /

Concentrated polymer solutions and melts show some interesting rheological phenomena such as non-Newtonian flow, the Weissenberg effect, and stress overshoot at the beginning of flow. Recently both experimental and theoretical investigations have been extensively developed on various nonlinear viscoelastic properties of these systems. Since Oldroyd's paper, ${ }^{1}$ many authors have given phenomenological considerations in order to construct constitutive equations to describe these properties. Recently Tanaka, Yamamoto, and Takano $^{2}$ have developed a molecular theory of non-Newtonian flow based upon a molecular model of Graessley, ${ }^{3}$ combined with the three-dimensional generalized Maxwell model proposed by Lodge ${ }^{4}$ and other authors. ${ }^{5}$ On the other hand, Yamamoto ${ }^{6}$ has proposed the so-called network theory based on a molecular model which is sufficiently general that it may be applicable to nonlinear viscoelasticity. In this paper we will investigate on the basis of this network theory the nonlinear viscoelastic properties of concentrated polymer systems, such as non-Newtonian viscosity and transient stress behaviors, as well as superpositions of a small oscillation upon a steady shearing flow.

According to Yamamoto's theory, the stress- deformation-time relation in integral form is given by

$$
\begin{aligned}
\boldsymbol{\sigma}(t) & =\int_{-\infty}^{t} \boldsymbol{\gamma}\left(t, t^{\prime}\right) \cdot \mathbf{T}\left(t, t^{\prime}\right) \cdot \boldsymbol{r}^{+}\left(t, t^{\prime}\right) \mathrm{d} t^{\prime} \\
\mathbf{T}\left(t, t^{\prime}\right)= & \sum_{N} \iiint(\boldsymbol{h} \boldsymbol{h})\left(\frac{1}{r} \frac{\mathrm{d} \Phi}{\mathrm{d} r}\right)_{r=\mid r\left(t, t^{\prime}\right) \boldsymbol{h l}} G\left(\boldsymbol{h}, N, \boldsymbol{t}^{\prime}\right) \\
& \times \exp \left[-\int_{t^{\prime}}^{t} \beta\left(\boldsymbol{r}\left(t^{\prime \prime}, t^{\prime}\right) \boldsymbol{h}, N\right) \mathrm{d} t^{\prime \prime}\right] \mathrm{d} \boldsymbol{h}(2)
\end{aligned}
$$

where $\boldsymbol{\sigma}(t)$ is the stress tensor at the time $t$, and $\gamma\left(t, t^{\prime}\right)$ is the relative deformation tensor at the time $t$ with respect to the system at the time $t^{\prime} .\left(\boldsymbol{\gamma}\left(t, t^{\prime}\right)=\boldsymbol{\gamma}(t) \cdot \boldsymbol{\gamma}^{-1}\left(t^{\prime}\right)\right.$ and the relative CauchyGreen strain tensor is given by $\mathrm{C}\left(t, t^{\prime}\right)=\boldsymbol{\gamma}^{+}\left(t, t^{\prime}\right)$. $\left.\boldsymbol{\gamma}\left(t, t^{\prime}\right)\right) . \quad G(\boldsymbol{h}, N, t)$ is the chain-reformation function, $\beta(\boldsymbol{h}, N)$ the chain-breakage coefficient, and $\Phi(|\boldsymbol{h}|, N, T)$ is the free energy of a chain $(N$ is the number of segments, $\boldsymbol{h}$ the end-to-end distance of the chain between two network junctions, and $T$ the absolute temperature). If any chain in the polymer network is represented by a Gaussian chain, its free energy is given by

$$
\Phi(|\boldsymbol{h}|, N, T)=\left(\frac{3 k T}{2}\right)\left(\frac{|\boldsymbol{h}|^{2}}{a^{2} N}\right)
$$

where $k$ is the Boltzmann constant and $a$ is the effective length of a segment. 


\section{THEORETICAL}

\section{Assumptions}

For the sake of simplicity in the calculation, the following assumption are used:

(1) The distribution of $N$ is neglected.

(2) Reformation of chains occurs in the natural state of the chains, i.e., the chainreformation function is given by

$$
\boldsymbol{G}(\boldsymbol{h}, N, t)=G_{0} \exp \left(-\frac{3 h^{2}}{2 N a^{2}}\right)
$$

(3) The probability of chain-breakage is proportional to the square of the end-to-end distance, i.e.,

$$
\beta(\boldsymbol{h}, N)=\left(\frac{\beta_{0}}{N a^{2}}\right) h^{2}
$$

Assumption (3) is essential to our calculation: it gives the nonlinearity of the mechanical properties. This functional form is presumed for the convenience of calculation. The main results obtained are not essentially affected by such a assumption.

\section{Relaxation Spectrum in the Equilibrium State}

We consider the periodic simple shear deformation with the deformation tensor

$$
\boldsymbol{\gamma}(t)=\left[\begin{array}{ccc}
1 & \gamma \mathrm{e}^{i \omega t} & 0 \\
0 & 1 & 0 \\
0 & 0 & 1
\end{array}\right](\gamma \ll 1)
$$

where $\omega$ is the frequency and $i=\sqrt{-1}$. Using the above-mentioned three assumptions the complex rigidity is obtained from eq 1,2 , and 6 :

$$
\begin{aligned}
G^{*}(i \omega)= & \frac{\sigma_{12}}{\gamma}=\int_{0}^{\infty} \beta_{0}^{-7 / 2} \tau^{-5 / 2}\left(2 \beta_{0} \tau+3\right) \\
& \times \exp \left(\frac{-3}{2 \beta_{0} \tau}\right) \frac{i \omega \tau}{1+i \omega \tau} \frac{\mathrm{d} \tau}{\tau}
\end{aligned}
$$

where

$$
\tau=\frac{N a^{2}}{\beta_{0} h^{2}}
$$

The relaxation spectrum, therefore, is given by

$$
H(\tau)=\beta_{0}^{-7 / 2} \tau^{-5 / 2}\left(2 \beta_{0} \tau+3\right) \exp \left(\frac{-3}{2 \beta_{0} \tau}\right)
$$

This spectrum is wedge-shaped, because we consider only the distribution of the end-to-end distance of the chain in the equilibrium network structure. Although there are some experimental data showing that the spectrum is rather wedgeshaped over a wide range of relaxation times, many experiments give a typical box-type spectra. We assume the distribution of $\beta_{0}$ to be constant for $\beta_{0}$ larger than $(1 / c) M^{-3.4}$ and otherwise zero, where $M$ is the molecular weight and $c$ is an arbitrary constant. This assumption means that the spectrum at zero shear rate has a plateau region corresponding to the intermolecular relaxation and that the maximum relaxation time is proportional to the 3.4th power of the molecular weight. Of course, this is a over-simplified and rather arbitrary assumption for the distribution of the "strength", of the entanglements and this assumption should be checked by a suitable molecular theoretical consideration after developing the molecular model for the chain entanglement: Unfortunately, we have not yet obtained such a powerful molecular model for the entanglement and in this treatment we will assume the above distribution. This assumption will have to be somewhat improved when the effect of the molecular weight distribution on the entanglement is discussed.

Thus we use the following relaxation spectrum in the equilibrium state:

$$
H(\tau, 0)=\int_{M-3.4 / c}^{1 / c} H(\tau) \mathrm{d} \beta_{0}
$$

The result of the numerical calculation is shown in Figure 1.

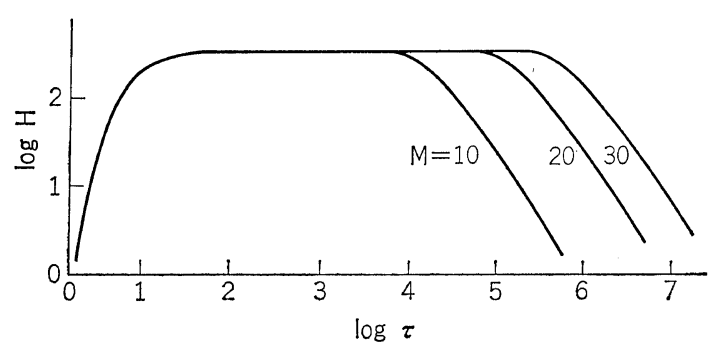

Figure 1. Relaxation spectra in the equilibrium state for various molecular weights.

\section{Non-Newtonian Viscosity}

Let the bulk of a given system flow in the $x$ direction with a constant gradient $\dot{\gamma}$ in the $y$ - 
direction. The strain tensor for this deformation is

$$
\gamma(t)=\left[\begin{array}{ccc}
1 & \dot{\gamma} t & 0 \\
0 & 1 & 0 \\
0 & 0 & 1
\end{array}\right]
$$

Substituting eq 11 into eq 1 and performing some elementary calculations, we can get the steady viscosity:

$$
\eta(\dot{\gamma})=\frac{\sigma_{12}}{\dot{\gamma}}=\int_{M^{-3.4 / c}}^{1 / c} \int_{0}^{\infty} \frac{B}{\dot{\gamma} D \sqrt{A D}} \mathrm{~d} \beta_{0} \mathrm{~d} \delta
$$

where

$$
\begin{aligned}
& A=3 / 2+\beta_{0} \delta \\
& B=\dot{\gamma} \delta\left(3 / 2+\beta_{0} \delta / 2\right) \\
& C=\dot{\gamma}^{2} \delta^{2}\left(3 / 2+\beta_{0} \delta / 3\right) \\
& D=A(A+C)-B^{2}
\end{aligned}
$$

This non-Newtonian viscosity is shown in Figure 2.

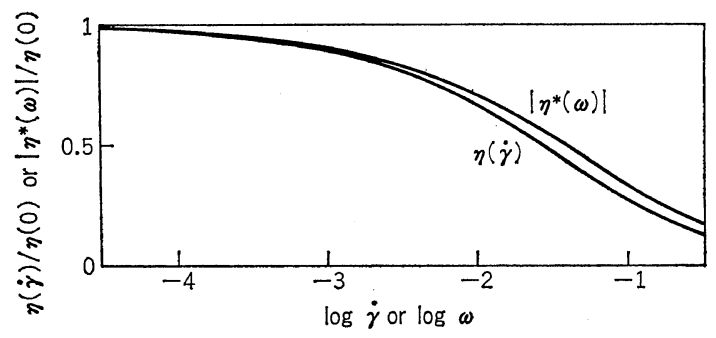

Figure 2. Steady viscosity and complex viscosity.

As has been experimentally pointed out by Onogi, et al., ${ }^{7,8}$ the shear-rate dependence of the steady viscosity is similar to the frequency dependence of the absolute value of the complex viscosity. In order to examine this relation, the absolute value of the complex viscosity is calculated for the relaxation spectrum of eq 10 . We obtain the following results:

$$
\begin{gathered}
\left|\eta^{*}\right|=\left(\eta^{\prime 2}+\eta^{\prime \prime 2}\right)^{1 / 2} \\
\eta^{\prime}=\int_{0}^{\infty} \frac{1}{\omega}\left(\tan ^{-1} y\right) \sqrt{x}(1+x) \exp (-x) \mathrm{d} x \\
\eta^{\prime \prime}=\int_{0}^{\infty} \frac{1}{2 \omega}\left(\log \left(1+y^{2}\right)\right) \sqrt{x}(1+x) \exp (-x) \mathrm{d} x \\
y=\frac{3 \omega c M^{3.4}}{2 x}
\end{gathered}
$$

The $\left|\eta^{*}\right| v s . \omega$ plot is also shown in Figure 2.
The $\eta(\dot{\gamma}) v s . \dot{\gamma}$ curve coincides well with the $\left|\eta^{*}\right|$ vs. $\omega$ curve.

It is a well-known experimental result that the steady-state, zero shear viscosity is proportional to the 3.4th power of the molecular weight $M$ of the polymer if the molecular weight is larger than the critical value $M_{\mathrm{c}}$. Furthermore, for a finite shear rate, there exists a second critical molecular weight $M_{\mathrm{c}}{ }^{\prime}\left(>M_{\mathrm{c}}\right)$, and with the larger molecular weight the gradient of the $\log \eta v s . \log M$ plot becomes again smaller than 3.4. As the shear rate increases, $M_{\mathrm{c}}{ }^{\prime}$ decreases. Such a dependence can be well explained by our model. In fact, from eq 12 and 13 we can obtain the $\dot{\gamma}$ dependence of the $\eta v s . M$ relation. The result of the numerical calculation is shown

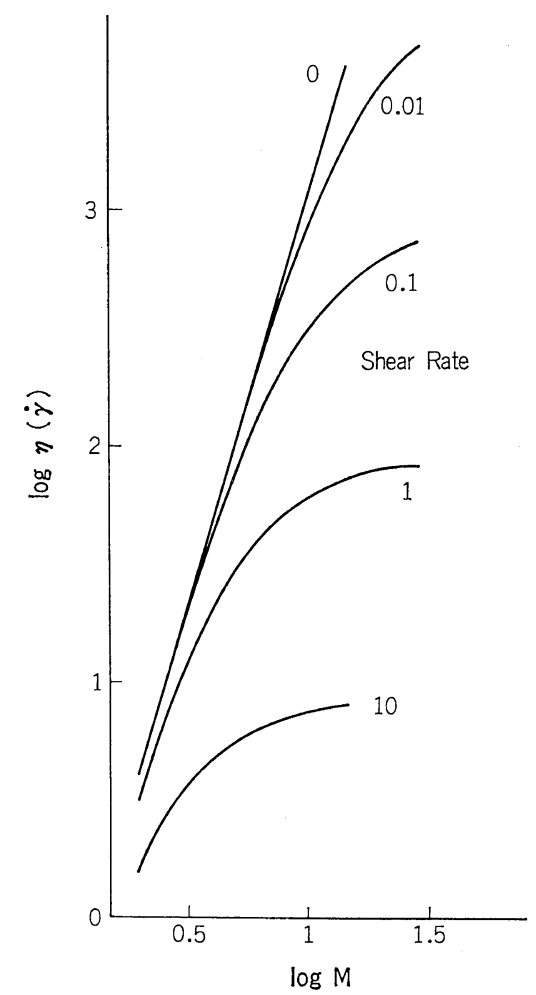

Figure 3. Steady viscosity vs. molecular weight relations for various rates of shear.

in Figure 3, which reproduces well the experimental results.

Transient Stress Change with Viscometric Flow Among the interesting properties connected 
with the flow behavior of concentrated polymer systems are the transient stress changes reported by several authors. ${ }^{9-14}$ The phenomenon of stress overshoot at the start of a viscometric shear flow is an example of such transient flow behavior. In the case of the stress overshoot experiment, the flow field, eq 11, is suddenly applied at the time $t=0$. From eq 1 we have the shear stress $\sigma_{\mathrm{s}}=\sigma_{12}$ and the normal stress $\Delta_{1} \sigma_{\mathrm{n}}=\sigma_{11}-\sigma_{22}$ at $t>0$.

$$
\begin{aligned}
& \sigma_{\mathrm{S}}=\int_{0}^{2 \pi}\left(S_{012}+\dot{\gamma} t S_{022}\right)\left[X\left(\beta_{0}\right)\right]_{M-3.4 / c}^{1 / c} \mathrm{~d} \phi \\
& +\int_{M-3.4 / c}^{1 / c} \int_{0}^{t}\left(T_{12}+\dot{\gamma} \delta T_{22}\right) \mathrm{d} \beta_{0} \mathrm{~d} \delta \\
& \Delta_{1} \sigma_{\mathrm{n}}=\int_{0}^{2 \pi}\left(S_{011}-S_{022}+2 \dot{\gamma} t S_{012}+\dot{\gamma}^{2} t^{2} S_{022}\right) \\
& \left.\times\left[X\left(\beta_{0}\right)\right]_{M}^{1 / c}-3.4 / c\right) \mathrm{d} \phi+\int_{M^{-3.4 / c}}^{1 / c} \int_{0}^{t}\left(T_{11}-T_{22}\right. \\
& \left.+2 \dot{\gamma} \delta T_{12}+\dot{\gamma}^{2} \delta^{2} T_{22}\right) \mathrm{d} \beta_{0} \mathrm{~d} \delta
\end{aligned}
$$

where

$$
\begin{gathered}
S_{011}=\frac{1+\cos \phi}{2 \pi}, \quad S_{012}=\frac{\sin \phi}{2 \pi}, \\
S_{022}=\frac{1-\cos \phi}{2 \pi} \\
T_{11}=\frac{A+C_{0}}{D_{0} \sqrt{A D_{\mathrm{o}}}}, \quad T_{12}=\frac{-B_{\mathrm{o}}}{D_{\mathrm{o}} \sqrt{A D_{\mathrm{o}}}}, \\
T_{22}=\frac{A}{D_{\mathrm{o}} \sqrt{D_{\mathrm{o}}}} \\
X\left(\beta_{0}\right)=P-\frac{2}{3 F \sqrt{F}} \tan ^{-1} \sqrt{\frac{F}{E}} \\
-\frac{1}{3} \sqrt{Q}(Q-2) \log \frac{\sqrt{E Q}+1}{\sqrt{E Q}-1}(F>0) \\
P-\frac{1}{3 F \sqrt{F}} \log \frac{\sqrt{E}+\sqrt{-F}}{\sqrt{E}-\sqrt{-F}} \\
+\frac{2}{3} \sqrt{-Q(Q-2)} \tan ^{-1} \sqrt{-E Q}(F<0) \\
P=\frac{4}{9} \frac{\sqrt{E}}{F}-\frac{4}{9} \sqrt{\frac{2}{3}} \log \frac{\sqrt{E}+\sqrt{\frac{3}{2}}}{\sqrt{E}-\sqrt{\frac{3}{2}}} \\
2\left(E+F-\frac{3}{2}\right) \\
\frac{3 F}{2}
\end{gathered}
$$

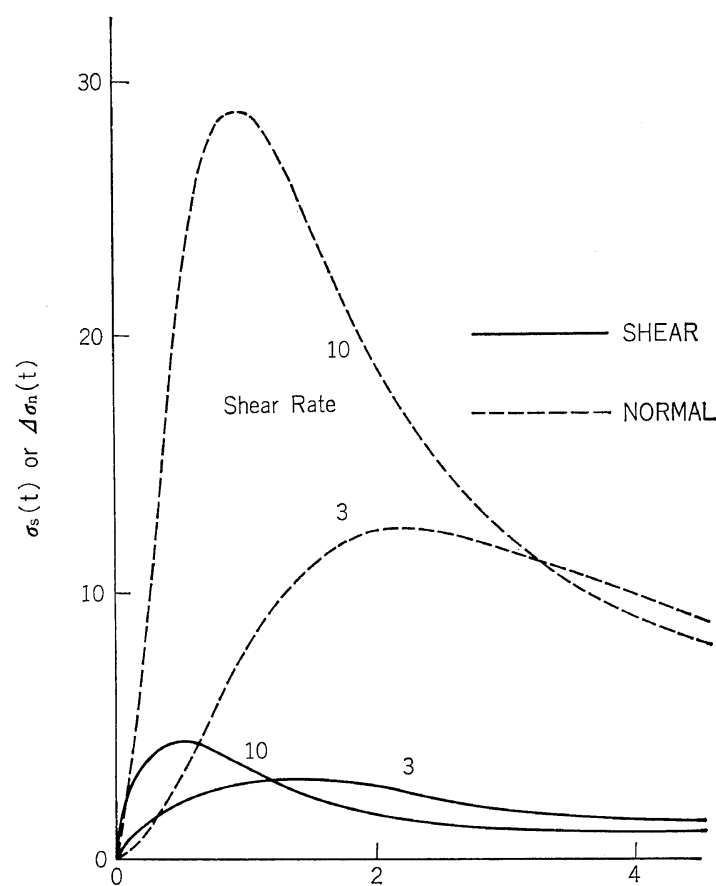

TIME

Figure 4. Initial stress overshoot for various rates of shear.

$$
B_{0}=\frac{\beta_{0} \dot{\gamma} \delta^{2}}{2}, \quad C_{0}=\frac{\beta_{0} \dot{\gamma}^{2} \delta^{3}}{3}, \quad D_{0}=A\left(A+C_{0}\right)-B_{0}{ }^{2}
$$

$$
\begin{aligned}
& E=\frac{3}{2}+\beta_{0} t, \\
& F=\frac{1}{2} \beta_{0} \dot{\gamma} t^{2}\left[\frac{1}{3} \dot{\gamma} t(1-\cos \phi)+\sin \phi\right]
\end{aligned}
$$

(22) The results of the numerical calculations of eq 18 and 19 are in Figure 4 . The higher the velocity gradient, the steeper the maximum at a shorter time from the start of flow. The maximum of the normal stress in general occurs later than that of the shear stress. This is agreement with the experimental results.

The stress relaxation after the stoppage of flow is another interesting transient phenomenon. Contrary to the overshoot, a simple shearing flow is suddenly removed at $t=0$. Using eq 1 , we can obtain the following stresses:

$$
\sigma_{\mathrm{s}}=\int_{M-3.4 / c}^{1 / c} \int_{0}^{\infty} \frac{B}{D_{\mathrm{r}} \sqrt{A_{\mathrm{r}} D_{\mathrm{r}}}} \mathrm{d} \beta_{0} \mathrm{~d} \delta
$$




$$
\Delta_{1} \sigma_{\mathrm{n}}=\int_{M-3.4 / c}^{1 / c} \int_{0}^{\infty} \frac{C}{D_{\mathrm{r}} \sqrt{A_{\mathrm{r}} D_{\mathrm{r}}}} \mathrm{d} \beta_{0} \mathrm{~d} \delta
$$

where

$$
A_{\mathrm{r}}=\frac{3}{2}+\beta_{0} \delta+\beta_{0} t, \quad D_{\mathrm{r}}=A_{\mathrm{r}}\left(A_{\mathrm{r}}+C\right)-B^{2}
$$

These equations give a longer apparent relaxation time for the normal stress $t_{\mathrm{r}}{ }^{\mathrm{n}}$ than that for the shear stress $t_{\mathrm{r}}^{\mathrm{s}}$, and both $t_{\mathrm{r}}^{\mathrm{n}}$ and $t_{\mathrm{r}}^{\mathrm{s}}$ are decreasing functions of the shear rate, as shown in Figure 5. Experimental results show the same tendency.

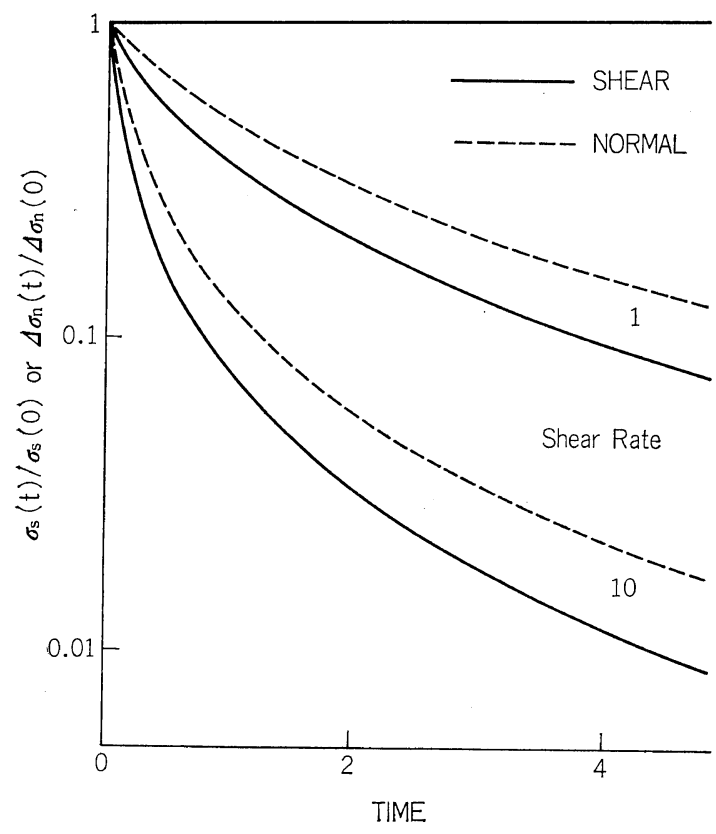

Figure 5. Stress relaxation after stoppage of flow for various rates of shear.

\section{Stress Relaxation under Large Deformation}

Besides the rate dependences of the viscoelastic processes, their deformation dependence should provide important informations on the nature of nonlinear viscoelastic phenomena. Stress relaxation under large deformation is an interesting transient phenomenon from this point of view. Einaga, et al., ${ }^{15}$ measured the relaxation moduli of polymer solutions under torsional deformation and found a strong deformation dependence. Our proposed model enables us to treat this stress relaxation. We consider a large shear deformation with a strain tensor

$$
\gamma(t)=\left[\begin{array}{lll}
1 & \gamma & 0 \\
0 & 1 & 0 \\
0 & 0 & 1
\end{array}\right](t>0)
$$

instantaneously applied to the system at time $t=0$. Using eq 1 we can get the following deformation-dependent shear relaxation modulus:

$$
G(t, \gamma)=\int_{0}^{2 \pi}\left(\frac{\sin \dot{\phi}}{\gamma}+1-\cos \phi\right)\left[X\left(\beta_{0}\right)\right]_{M L}^{1 / c}-3.4 / c d \phi
$$

In this case, in $X\left(\beta_{0}\right)$, instead of the $F$ of eq 26 we should use the following expression:

$$
F=\beta_{0} t \gamma\left[\frac{\gamma(1-\cos \phi)}{2}+\sin \phi\right]
$$

In Figure 6 is shown the result of the numerical calculation of the shear modulus for various degrees of deformations. This is in qualitative agreement with the experimental results of Einaga, et al.

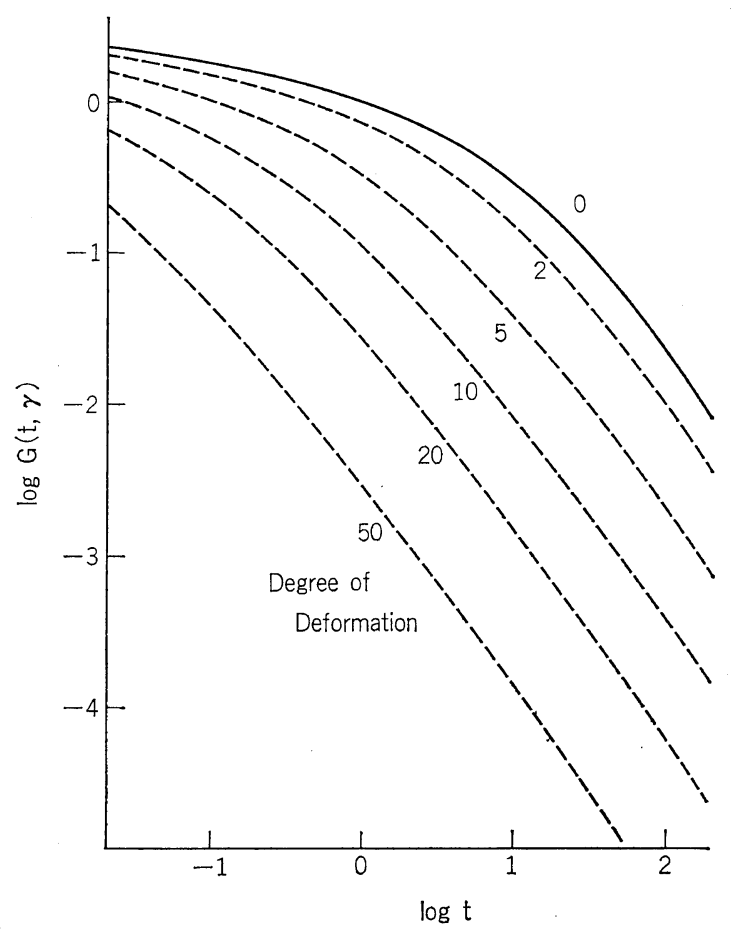

Figure 6. Ordinary stress relaxation for large stepwise deformation. 


\section{Y. TAKANO}

Small Oscillations Superimposed on Steady Shearing Flow

Another interesting form of deformation is a superposition of a small oscillatory motion on steady shearing flow. One form is a "parallel" superposition, and another is an "orthogonal", superposition. The details of geometry of these superpositions are given in Yamamoto's paper, ${ }^{18}$ and we can omit them here. The first careful experimental observation of parallel superposition was made by Osaki, et al., ${ }^{16}$ while a theoretical consideration of superposed parallel oscillation has been given by several authors. ${ }^{4,17}$ Recently Yamamoto treated both the parallel and orthogonal cases by the use of the concept of a deformation-rate-dependent relaxation spectrum in his phenomenological considerations. ${ }^{18}$ In this section let us consider deformations in this category.

In the case of parallel superposition, the deformation tensor is

$$
\gamma(t)=\left[\begin{array}{ccc}
1 & \dot{\gamma} t+a_{0} \mathrm{e}^{i \omega t} & 0 \\
0 & 1 & 0 \\
0 & 0 & 1
\end{array}\right]
$$

It is assumed that the amplitude $a_{0}$ of the superposed oscillation is so small that higher terms than $a_{0}$ can be neglected and thus only linear terms will be treated. From eq 1 the shear stress can be calculated with the following result:

$$
\sigma_{\mathrm{s}}=\sigma_{12}=\sigma_{21}=\eta(\dot{\gamma}) \dot{\gamma}+G_{\|}{ }^{*}(\omega) a_{0} \mathrm{e}^{i \omega t}
$$

where $\eta(\dot{\gamma})$ is the steady viscosity given by eq 12 and $G_{\|}{ }^{*}(\omega)=G_{\|}{ }^{\prime}(\omega)+i G_{\|}{ }^{\prime \prime}(\omega)$ is the apparent complex modulus:

$$
\begin{aligned}
G_{\|}{ }^{\prime}(\omega) & =\int \mathrm{d} \beta_{0} \int_{0}^{\infty} \frac{\Phi_{\mathrm{p}}{ }^{\prime}}{D^{2} \sqrt{A D}} \mathrm{~d} \delta \\
G_{\|}{ }^{\prime \prime}(\omega) & =\int \mathrm{d} \beta_{0} \int_{0}^{\infty} \frac{\Phi_{\mathrm{p}}{ }^{\prime \prime}}{D^{2} \sqrt{A D}} \mathrm{~d} \delta
\end{aligned}
$$

Here $\Phi_{\mathrm{p}}{ }^{\prime}$ and $\Phi_{\mathrm{p}}{ }^{\prime \prime}$ are given by the following expressions:

$$
\begin{aligned}
\Phi_{\mathrm{p}}{ }^{\prime}= & {\left[\frac{3}{2}(1-\cos (\omega \delta))-\frac{\beta_{0}}{\omega} \sin (\omega \delta)+\beta_{0} \delta\right] } \\
& \times\left[-\frac{2}{3} \beta_{0}{ }^{2} \dot{\gamma}^{2} \delta^{4}-\frac{11}{4} \beta_{0} \dot{\gamma}^{2} \delta^{3}+\left(\beta_{0} \delta+\frac{3}{2}\right)^{2}\right] \\
& -\frac{3 \beta_{0} \dot{\gamma}^{2} \delta}{2 \omega^{2}}(1-\cos (\omega \delta))\left[\frac{9}{2}+\frac{9}{2} \beta_{0} \delta+\beta_{0}{ }^{2} \delta^{2}\right]
\end{aligned}
$$

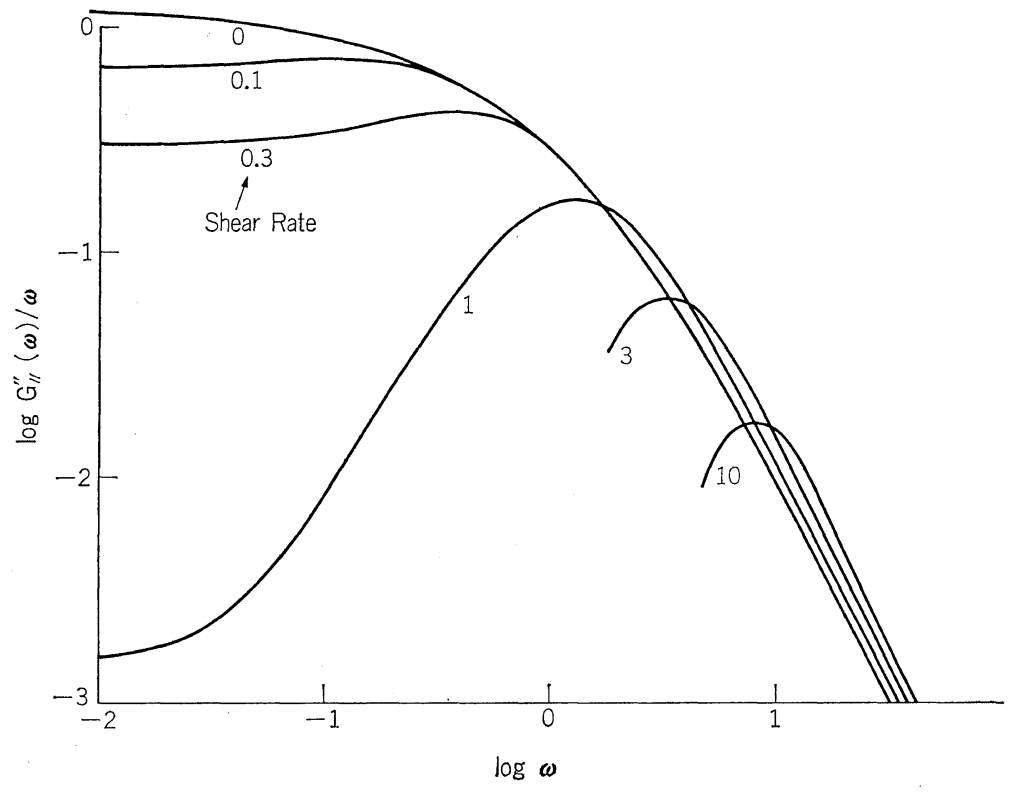

Figure 7. Dynamic viscosity as a function of frequency for various superposed rates of shear in parallel superposition. 
Network Theory for Nonlinear Viscoelasticity

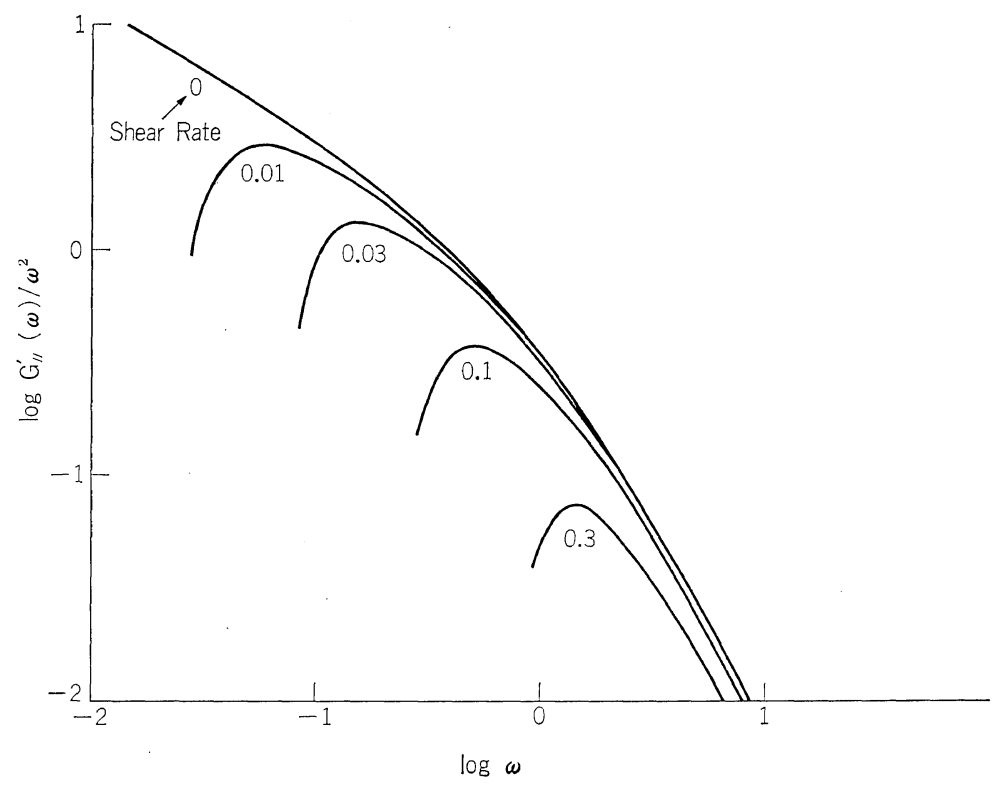

Figure-8. Quantity $G_{\|}{ }^{\prime}(\omega) / \omega^{2}$ as a function of frequency for various superposed rates of shear in parallel superposition.

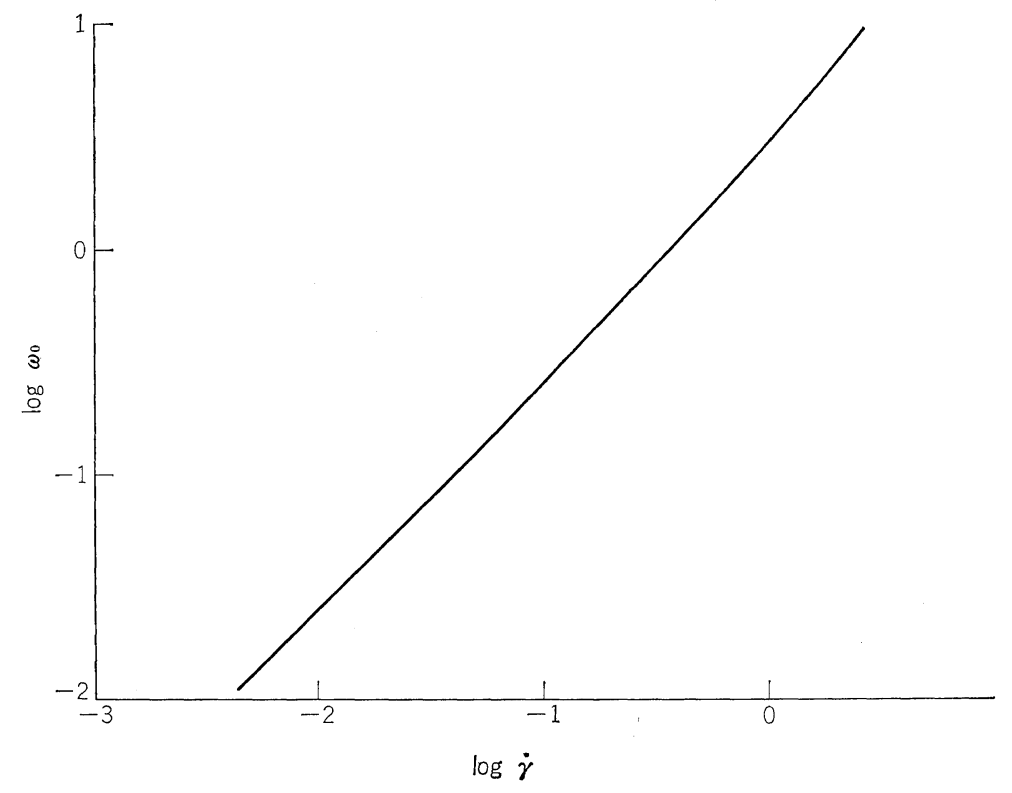

Figure 9. Frequency at which the apparent dynamic modulus vanishes as a function of superposed rate of shear in parallel superposition. 


\section{Y. TAKANO}

$$
\begin{aligned}
\Phi_{\mathrm{p}}{ }^{\prime \prime}= & {\left[\frac{3}{2} \sin (\omega \delta)+\frac{\beta_{0}}{\omega}(1-\cos (\omega \delta))\right] } \\
& \times\left[-\frac{2}{3} \beta_{0}{ }^{2} \dot{\gamma}^{2} \delta^{4}-\frac{11}{4} \beta_{0} \dot{\gamma}^{2} \delta^{3}+\left(\beta_{0} \delta+\frac{3}{2}\right)^{2}\right] \\
& -\frac{3 \beta_{0} \dot{\gamma}^{2} \delta}{2 \omega^{2}}(\omega \delta-\sin (\omega \delta)) \\
& \times\left[\frac{9}{2}+\frac{9}{2} \beta_{0} \delta+\beta_{0}{ }^{2} \delta^{2}\right]
\end{aligned}
$$

The values of $G_{\|}{ }^{\prime}(\omega) / \omega^{2}$ and $G_{\|}{ }^{\prime \prime}(\omega) / \omega$ are plotted logarithmically as functions of the frequency for some fixed values of the superimposed steady shear rate in Figures 7 and 8. The values of the dynamic moduli $G_{\|}{ }^{\prime}(\omega)$ become negative at small oscillatory frequencies. The frequency at which the apparent dynamic modulus vanishes and the phase difference $\delta$ $\left(\tan \delta=G^{\prime \prime} / G^{\prime}\right)$ just equals $\pi / 2$ is denoted $\omega_{0}$. Figure 9 shows that the critical frequency $\omega_{0}$ is approximately proportional to the superimposed steady shear rate $\dot{\gamma}$. Booij ${ }^{19}$ and Kataoka and $\mathrm{Ueda}^{20}$ have found in their experiments on the parallel superpositions a simple relation between the superimposed shear rate and the critical frequency: $\omega_{0}=\dot{\gamma} / 2$. These results are parallel to the present ones.
For the orthogonal case, the deformation tensor is assumed to be

$$
\gamma(t)=\left[\begin{array}{ccc}
1 & \dot{\gamma} t & 0 \\
0 & 1 & 0 \\
0 & b_{0} \mathrm{e}^{i \omega t} & 1
\end{array}\right]
$$

Making use of the assumption of small oscillations, $b_{0} \ll 1$, we find the shear stress for the oscillation to be

$$
\sigma_{\mathrm{s}}=\sigma_{32}=\sigma_{23}=G_{\perp}{ }^{*}(\omega) b_{0} \mathrm{e}^{i \omega t}
$$

with the orthogonal complex modulus $G_{\perp}{ }^{*}(\omega)=$ $G_{\perp}{ }^{\prime}(\omega)+i G_{\perp}{ }^{\prime \prime}(\omega)$ :

$$
\begin{aligned}
G_{\perp}{ }^{\prime}(\omega) & =\int \mathrm{d} \beta_{0} \int_{0}^{\infty} \frac{\Phi_{\circ}{ }^{\prime}}{A D \sqrt{A D}} \mathrm{~d} \delta \\
G_{\perp}{ }^{\prime \prime}(\omega) & =\int \mathrm{d} \beta_{0} \int_{0}^{\infty} \frac{\Phi_{\circ}{ }^{\prime \prime}}{A D \sqrt{A D}} \mathrm{~d} \delta
\end{aligned}
$$

Here

$$
\begin{aligned}
& \Phi_{\circ}^{\prime}=\left[\frac{3}{2}(1-\cos (\omega \delta))-\frac{\beta_{0}}{\omega} \sin (\omega \delta)+\beta_{0} \delta\right](A+C) \\
& \Phi_{\circ}{ }^{\prime \prime}=\left[\frac{3}{2} \sin (\omega \delta)+\frac{\beta_{0}}{\omega}(1-\cos (\omega \delta))\right](A+C)
\end{aligned}
$$

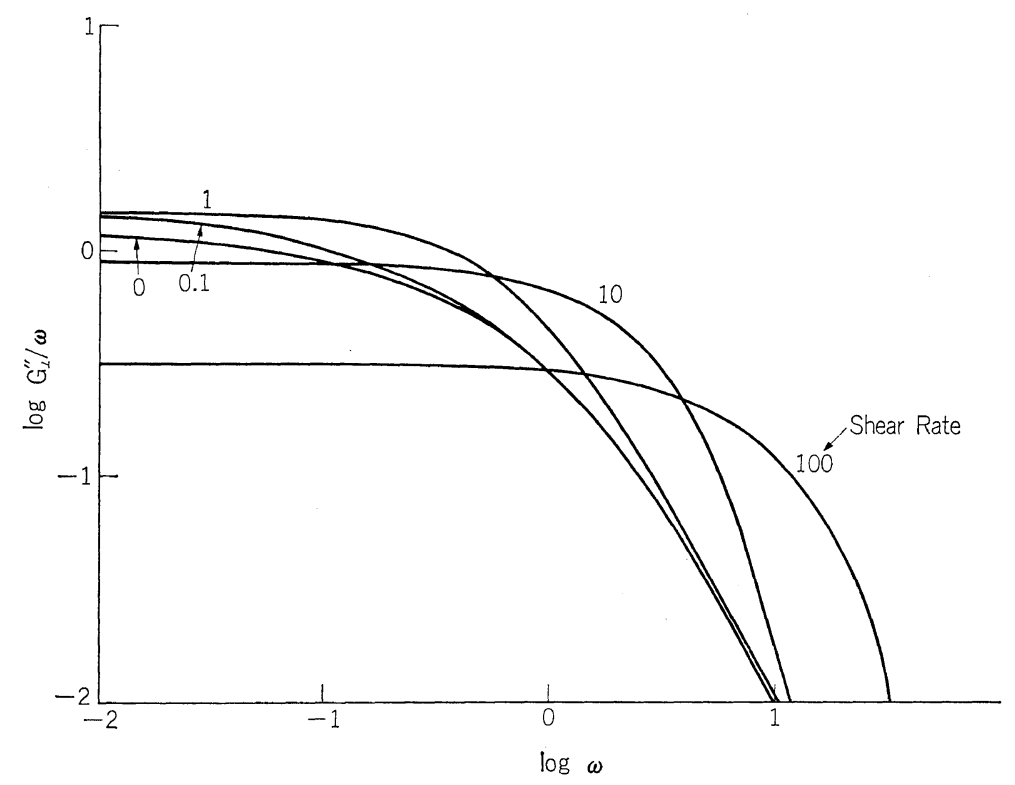

Figure 10. Dynamic viscosity as a function of frequency for various superposed rates of shear in orthogonal superposition. 


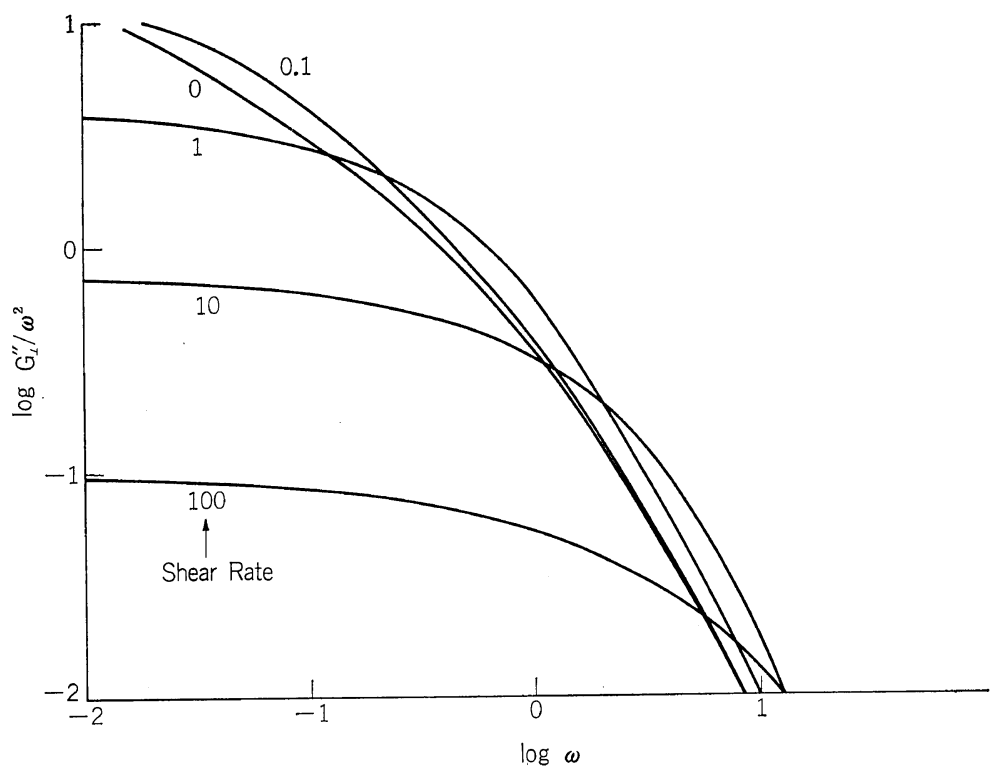

Figure 11. Quantity $G_{\perp}^{\prime}(\omega) / \omega^{2}$ as a function of frequency for various superposed rates of shear in orthogonal superposition.

An experiment on the superposed orthogonal oscillation was carried out by Simmons. ${ }^{21}$ In this case the experimentally observed curve of the complex modulus behaved just as that of the complex modulus in linear viscoelasticity and had no negative region, as is predicted in the present calculation. In Figures 10 and 11 the $\log G_{\perp}{ }^{\prime}(\omega) / \omega^{2} v s . \log \omega$ curve and the $\log G_{\perp}{ }^{\prime \prime}(\omega) / \omega$ $v s . \log \omega$ curve are shown. Figure 11 predicts that there exists a frequency region where the dynamic viscosity $\eta^{\prime}(\omega)$ increases with the increase of the superimposed shear rate for a fixed frequency. This tendency is also found in parallel superpositions of the present model, as is shown in Figure 8, though it is not so clear. This behavior was not found in the experimental results of Simmons; ${ }^{21}$ however, this behavior is not an unreasonable phenomenon considering the fact that the peak appearing in the $G_{\perp}{ }^{\prime \prime}(\omega) v s . \omega$ curve shifts to larger $\omega$ upon increasing the superposed shear rate. According to the general theory of viscoelasticity the peak shifts toward higher $\omega$ as the temperature increases and the apparent relaxation time becomes shorter. In the present case the increase of shear rate may be considered to induce a more activated condition of molecular motion, namely an increase of temperature. In calculations of the parallel and orthogonal superpositions, for the sake of simplicity the integration over $\beta_{0}$ is not performed and the results are shown for the case of $\beta_{0}=1$. This simplification will not significantly affect the final results.

In addition to this oscillatory shear stress and the steady shear stress $\sigma_{12}=\sigma_{21}=\eta(\dot{\gamma}) \dot{\gamma}$, we have another cross shear stress

$$
\sigma_{\mathrm{s}}{ }^{\prime}=\sigma_{31}=\sigma_{13}=F^{*}(\omega) b_{0} \dot{\gamma} \mathrm{e}^{i \omega t}
$$

The "complex cross modulus" $F^{*}(\omega)=F^{\prime}(\omega)+$ $i F^{\prime \prime}(\omega)$ is expressed as

$$
\begin{aligned}
& F^{\prime}(\omega)=\int \mathrm{d} \beta_{0} \int_{0}^{\infty} \frac{\Psi^{\prime}}{A D \sqrt{A D}} \mathrm{~d} \delta \\
& F^{\prime \prime}(\omega)=\int \mathrm{d} \beta_{0} \int_{0}^{\infty} \frac{\Psi^{\prime \prime}}{A D \sqrt{A D}} \mathrm{~d} \delta
\end{aligned}
$$

where

$$
\begin{aligned}
& \Psi^{\prime}=\left[\frac{3}{2}(1-\cos (\omega \delta))-\frac{\beta_{0}}{\omega} \sin (\omega \delta)+\beta_{0} \delta\right] B \\
& \Psi^{\prime \prime}=\left[\frac{3}{2} \sin (\omega \delta)+\frac{\beta_{0}}{\omega}(1-\cos (\omega \delta))\right] B
\end{aligned}
$$

As has been pointed out in Yamamoto's paper, ${ }^{18}$ 
an experimental determination of this cross component may be very difficult.

Rate-Dependent and Strain-Dependent Relaxation Spectra

As has been pointed by Yamamoto, ${ }^{18}$ the concept of a rate-of-deformation-dependent relaxation spectrum is useful for the analysis of nonlinear viscoelasticity. Using the present model, we can calculate the rate-dependent relaxation spectra $H_{\mathrm{s}}(\tau, \dot{\gamma})$ from the shear component and $H_{\mathrm{n}}(\tau, \dot{\gamma})$ from the normal component of stress relaxation after cessation of a steady shearing flow. The results are

$$
\begin{aligned}
& H_{\mathrm{S}}(\tau, \dot{\gamma})=\frac{1}{\dot{\gamma} \tau^{7 / 2}} \int_{M^{-3.4 / c}}^{1 / c} \frac{1}{\beta_{0}^{5 / 2}} \int_{0}^{\infty} \int_{0}^{\pi} \int_{0}^{2 \pi} \\
& \times \sin ^{3} \theta \sin \phi \cos \phi \exp \left(-\frac{q}{\tau}\right) \mathrm{d} \beta_{0} \mathrm{~d} \delta \mathrm{d} \theta \mathrm{d} \phi \\
& H_{\mathrm{n}}(\tau, \dot{\gamma})=\frac{1}{2 \dot{\gamma}^{2} \tau^{9 / 2}} \int_{M^{-3.4 / c}}^{1 / c} \frac{1}{\beta_{0}^{5 / 2}} \int_{0}^{\infty} \int_{0}^{\pi} \int_{0}^{2 \pi} \\
& \times \sin ^{3} \theta\left(\cos ^{2} \phi-\sin ^{2} \phi\right) \exp \left(-\frac{q}{\tau}\right) \mathrm{d} \beta_{0} \mathrm{~d} \delta \mathrm{d} \theta \mathrm{d} \phi
\end{aligned}
$$

where

$$
q=\frac{1}{\beta_{0}}\left[A-(2 B \cos \phi-C \sin \phi) \sin \theta \sin ^{2} \phi\right]
$$

In the limit of zero shear rate, both $H_{\mathrm{s}}(\tau, \dot{\gamma})$ and $H_{\mathrm{n}}(\tau, \dot{\gamma})$ tend to the equilibrium relaxation spectrum $H(\tau, 0)$. A rough estimate of $H_{\mathrm{s}}(\tau, \dot{\gamma})$ shows that the effect of an increase of the shear rate is cut off the spectrum in the long-relaxation-time region.

A strain-dependent relaxation spectrum is also calculated from the relaxation modulus under large deformation:

$$
\begin{aligned}
& H(\tau, \gamma)=\int_{M^{-3.4 / c}}^{1 / c} \frac{1}{\gamma \beta_{0}^{7 / 2} \tau^{3 / 2}} \int_{0}^{\pi} \int_{0}^{2 \pi} \\
& \quad \times \frac{1}{q^{\prime}} \sin ^{3} \theta \sin \phi \cos \phi \exp \left(-\frac{3 q^{\prime}}{2 \tau}\right) \mathrm{d} \beta_{0} \mathrm{~d} \theta \mathrm{d} \phi
\end{aligned}
$$

with

$$
q^{\prime}=\frac{1}{\beta_{0}}\left(1-2 \gamma \sin ^{2} \theta \sin \phi \cos \phi+\gamma^{2} \sin ^{2} \theta \sin ^{2} \phi\right)
$$

\section{DISCUSSIONS}

In the present work we can semiphenomenologically explain some of the nonlinear viscoelastic properties of concentrated polymer systems. The assumption that the probability of chain-breakage is proportional to the square of the end-to-end distance in a chain is essential to our theory and causes the nonlinearity of the mechanical properties, although this dependence on the end-to-end distance is rather arbitrary and we chose it in order to carry out exactly some complicated integrals. On the other hand, the effect of flow on the change of the rate of formation of entanglement is not taken into account in the theory. This effect has been previously treated explicitly by Graessley $^{3}$ and by the author, et al. ${ }^{2}$ All measurable quantities in the network theory may be derived from the knowledge of the distribution function $F(\boldsymbol{h}, N, t)$ of the network chains alone, and it may be very difficult to separate the effect of the chainreformation and that of the chain-breakage by the analysis of suitable experimental results. This problem in the treatment of chain entanglements still remains to be solved.

On the other hand, as compared with the phenomenological construction of constitutive equations, the present model has the advantage of being able to discuss the rate as well as the deformation dependence of the viscoelastic characteristic functions by the same model, which is rather difficult using the present constitutive equations.

The numerical calculations were performed on the HITAC 5020 and 8700 computers at the University of Tokyo.

Acknowledgment. The author wishes to express his sincere thanks to Professor Misazo Yamamoto of Tokyo Metropolitan University for his continuing encouragement and helpful discussions. The present investigation has been supported partly by the Scientific Research Funds of the Ministry of Education.

\section{REFERENCES}

1. J. G. Oldroyd, Proc. Roy. Soc. Ser. A, 200, 523 (1950).

2. T. Tanaka, M. Yamamoto, and Y. Takano, $J$. 
Macromol. Sci. (Phys.), B4, 931 (1970).

3. W. W. Graessley, J. Chem. Phys., 43, 2696 (1965).

4. A. S. Lodge, "Elastic Liquids", Academic Press, New York, N.Y., 1964.

5. K. Walters, Qurt. J. Mech. Appl. Math., 13, 444 (1960); ibid., 15, 63 (1962); Frederickson, Chem. Eng. Sci., 17, 155 (1962); M. Yamamoto, J. Phys. Soc. Japan, 25, 239 (1968).

6. M. Yamamoto, J. Phys. Soc. Japan, 11, 413 (1956).

7. S. Onogi, I. Hamana, and H. Hirai, J. Appl. Phys., 29, 1503 (1958).

8. W. P. Cox and E. H. Mers, J. Polym. Sci., 28, 619 (1958).

9. K. Komuro, Y. Todani, and N. Nagata, Polymer Letters, 2, 643 (1964).

10. G. V. Vinogradov and I. M. Belkin, J. Polym. Sci., Part A, 3, 917 (1965).

11. J. D. Huppler, I. F. MacDonald, E. Ashare, T. W. Spriggs, R. B. Bird, and L. A. Homes, Trans. Soc. Rheol., 11, 181 (1967).

12. N. Nishida, D. G. Salladay, and J. L. White, J. Appl. Polym. Sci., 15, 1181 (1971). Also see ref 22 .
13. M. Sakai, H. Fukaya, and M. Nagasawa, Trans. Soc. Rheol., 16, 635 (1972).

14. S. Kuroiwa and M. Nakamura, Zairyo, 15, 297 (1966); Kobunshi Kagaku (Chem. High Polymers), 24, 441 (1967).

15. Y. Einaga, K. Osaki, M. Kurata, S. Kimura, and M. Tamura, Polymer J., 2, 550 (1971).

16. K. Osaki, M. Tamura, and M. Kurata, J. Phys. Chem., 69, 4183 (1965).

17. I. F. MacDonald and R. B. Bird, ibid., 70, 2068 (1966); H. C. Booij, Rheol. Acta, 5, 222 (1966); R. I. Tanner, Trans. Soc. Rheol., 12, 155 (1968); H. Markovitz, Proc. Fifth Intern. Cong. Rheol., 1, 499 (1969).

18. M. Yamamoto, Trans. Soc. Rheol., 15, 331, 783 (1971).

19. H. C. Booij, Rheol. Acta, 5, 215 (1966).

20. T. Kataoka and S. Ueda, J. Polym. Sci., Part $A, 27,475$ (1969).

21. J. M. Simmons, J. Sci. Instrum., 43, 887 (1966); Rheol. Acta, 7, 184 (1968). Also see ref 23.

22. I-J. Chen and D. C. Bogue, Trans. Soc. Rheol., 16, 59 (1972).

23. R. I. Tanner and G. Williams, Rheol. Acta, 10, 528 (1971). 